\title{
Precision measurements of Standard Model parameters with the ATLAS detector
}

\author{
Aleksandra Dimitrievska* (On behalf of the ATLAS Collaboration) \\ Institute of Physics, Belgrade \\ E-mail: aleksandra.dimitrievska@cern.ch
}

\begin{abstract}
The ATLAS Collaboration is engaged in precision measurements of fundamental Standard Model parameters, e.g. the weak-mixing angle. A measurement of the forward-backward asymmetry for the neutral current Drell-Yan process is presented and the results are then used to extract a measurement of the effective weak mixing angle. This measurement shows significant sensitivity to the uncertainties of the parton density functions of the proton (PDFs). The status of the $W$ boson mass measurement preparations with ATLAS detector is described.
\end{abstract}

The European Physical Society Conference on High Energy Physics 22-29 July 2015

Vienna, Austria

* Speaker. 


\section{Introduction}

The Standard Model of particle physics provides a successful description of the fundamental particles and their interactions. The precision measurement of the Standard Model parameters are important part of ATLAS physics program at the LHC [1]. After the discovery of the Higgs boson at the LHC by the ATLAS and CMS experiments [2,3] and the precise measurement of the its mass with a value $m_{\mathrm{H}}=125.09 \pm 0.21$ (stat.) \pm 0.11 (syst.) $\mathrm{GeV}$ [4] the Standard Model becames over-constrained. Self-consistency of the Standard Model can be tested by performing precision measurements of its parameters and confronting the results with theoretical predictions.

Currently the most interesting set of constraints relates the Higgs boson mass $m_{\mathrm{H}}$ to $m_{\mathrm{W}}$ and to the top quark mass $m_{\mathrm{t}}$. Also, the electroweak mixing angle $\sin ^{2} \theta_{\mathrm{W}}$ and $W$ boson mass are connected at tree level as $\sin ^{2} \theta_{\mathrm{W}}=1-m_{\mathrm{W}}^{2} / m_{\mathrm{Z}}^{2}$, where $m_{\mathrm{Z}}$ is $Z$ boson mass, hence any inconsistency between these three measurements can give a hint for new physics. Figure 1 illustrates the scans of $m_{\mathrm{W}}$ vs $m_{\mathrm{t}}$ and $m_{\mathrm{W}} \mathrm{vs} \sin ^{2} \theta_{\text {eff }}^{\text {lept }}$ for the direct measurement compared to the results of the global fit of the electroweak sector of the Standard Model including and excluding the direct $m_{\mathrm{H}}$ measurement [5].
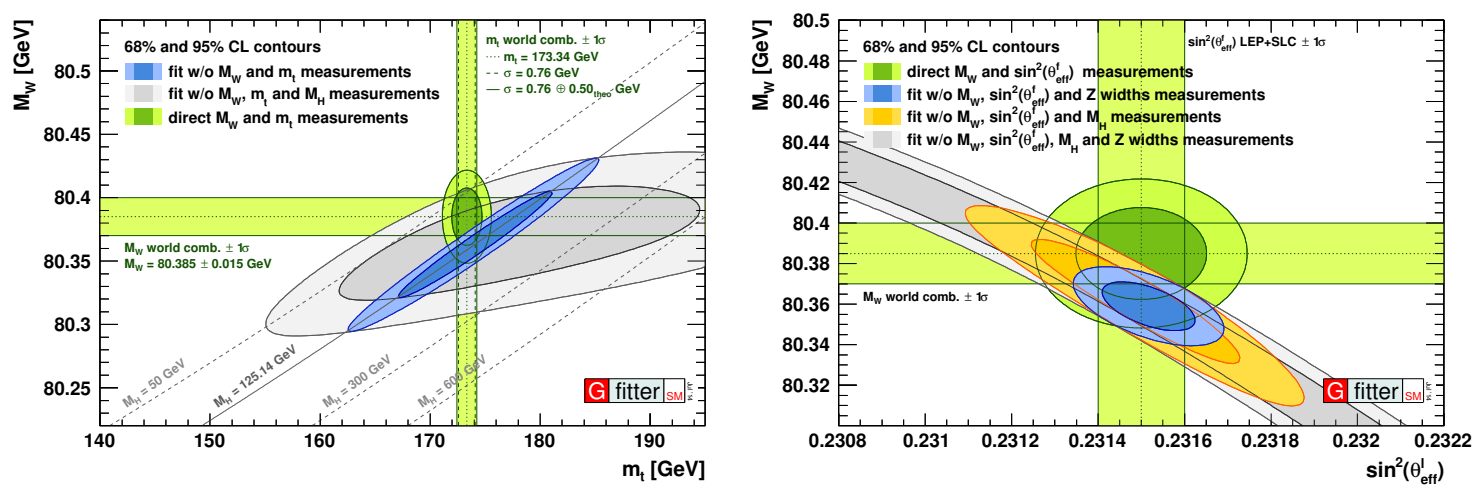

Figure 1: Contours at $68 \%$ and $95 \%$ CL obtained from scans of $m_{\mathrm{W}}$ versus $m_{\mathrm{t}}$ (left) and $m_{\mathrm{W}}$ versus $\sin ^{2} \theta_{\mathrm{eff}}^{\text {lept }}$ (right) compared to the direct measurements. [5].

These proceedings will focus on the measurement of the weak-mixing angle from the forwardbackward asymmetry of electron and muon pair-production and the status of the preparation for the $\mathrm{W}$ boson mass measurement with the ATLAS detector.

\section{Measurement of the forward-backward asymmetry of electron and muon pair-production}

The Drell-Yan process has been used for the measurement of the forward-backward asymmetry with the data recorded in 2011 using the proton-proton collisions with center-of-mass energy of $\sqrt{s}=7 \mathrm{TeV}$, which corresponds to an integrated luminosity of $4.8 \mathrm{fb}^{-1}$ [6]. The Drell-Yan process occurs when a quark from one of the colliding protons annihilates with an anti-quark from the other proton. In this process a virtual neutral gauge boson is created that decays to a pair of opposite charged leptons: $q \bar{q} \rightarrow Z / \gamma^{*} \rightarrow \ell^{+} \ell^{-}(\ell=e, \mu)$. Due to the V-A nature of the electroweak interaction, the leptons produced in this annihilation process have a forward-backward asymmetry $A_{\mathrm{FB}}$ with respect to the quark direction in the rest frame of the dilepton system, more precisely the 
Collins-Soper frame [7]. This asymmetry is observed in the polar angle $\theta$ between the final state leptons and the incoming quark direction:

$$
\cos \theta_{\mathrm{CS}}^{*}=\frac{p_{z, l l}}{\left|p_{z, l l}\right|} \frac{2\left(p_{1}^{+} p_{2}^{-}-p_{1}^{-} p_{2}^{+}\right)}{m_{l l} \sqrt{m_{l l}^{2}+p_{\mathrm{T}, l l}^{2}}},
$$

where $p_{i}^{ \pm}=\frac{1}{\sqrt{2}}\left(E_{i} \pm p_{i, z}\right) ; E$ and $p_{z}$ are the energy and longitudinal momentum of the leptons; $p_{z, l l}$, $m_{l l}, p_{\mathrm{T}, l l}$ are the longitudinal momentum, invariant mass and transverse momentum of the dilepton system.

The dilepton events having $\cos \theta_{\mathrm{CS}}^{*}>0$ are classified as forward $(\mathrm{F})$, while those having $\cos \theta_{\mathrm{CS}}^{*}<0$ are classified as backward (B). The sign of $\cos \theta_{\mathrm{CS}}^{*}$ is defined with respect to the direction of the incoming quark which is unknown in proton-proton collisions, therefore the forward direction is defined as the longitudinal boost of the resulting lepton pair. This presumption is causing a dilution of the observed asymmetry. The probability of misidentifying the quark direction decreases with increasing boost of the dilepton system, therefore the dilution is reduced for dileptons produced at large rapidities.

This analysis uses electrons in two regions of the detector ${ }^{1}$ : central $(|\eta|<2.47)$ and forward $(2.5<|\eta|<4.9)$. In order to maximise the sensitivity the measurement is performed in three channels: CC electron (both electrons are from central region), $\mathrm{CF}$ electron (one electron is in central and the other in the forward region) and the muon channel (restricted to $|\eta|<2.4$ ). Figure 2 shows $\cos \theta_{\mathrm{CS}}^{*}$ distribution of events in three channels, for CF electron channel the forwardbackward asymmetry is large enough to be observed directly from the plot.
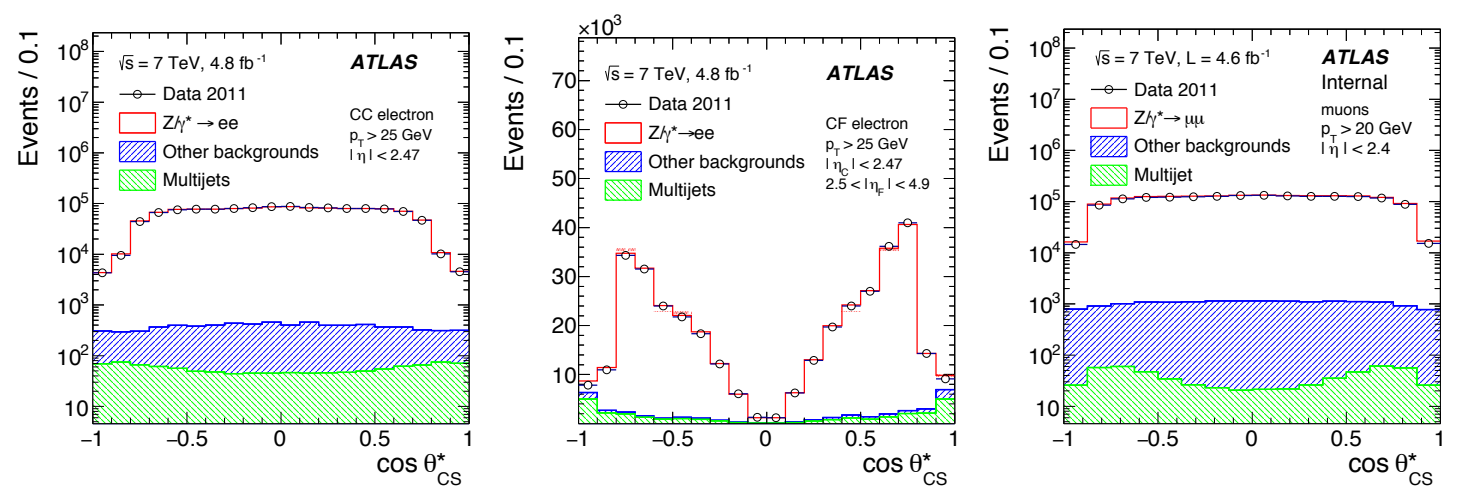

Figure 2: Distributions of $\cos \theta_{\mathrm{CS}}^{*}$ for $\mathrm{CC}, \mathrm{CF}$ electrons and muons. Data are shown by open circles and the total expectation is shown as a line [6].

The asymmetry is defined as:

$$
A_{\mathrm{FB}}=\frac{\sigma_{\mathrm{F}}-\sigma_{\mathrm{B}}}{\sigma_{\mathrm{F}}+\sigma_{\mathrm{B}}},
$$

where $\sigma_{\mathrm{F}}$ and $\sigma_{\mathrm{B}}$ are the cross sections for forward and backward event topologies. Detector level forward-backward asymmetry is shown on Figure 3. The asymmetry values are then unfolded from

\footnotetext{
${ }^{1}$ ATLAS uses a right-handed coordinate system with its origin at the nominal interaction point (IP) in the centre of the detector and the $z$-axis along the beam pipe. The $x$-axis points from the IP to the centre of the LHC ring, and the $y$-axis points upward. Cylindrical coordinates $(r, \varphi)$ are used in the transverse plane, $\varphi$ being the azimuthal angle around the beam pipe. The pseudorapidity is defined in terms of the polar angle $\theta$ as $\eta=-\ln \tan (\theta / 2)$.
} 
detector level to particle level to allow comparisons with theoretical predictions using an iterative Bayesian unfolding method [8]. The unfolding is done in two steps, first detector effects affecting kinematic distributions are unfolded, and in the second step corrections for dilution and acceptance are applied using the same unfolding procedure.
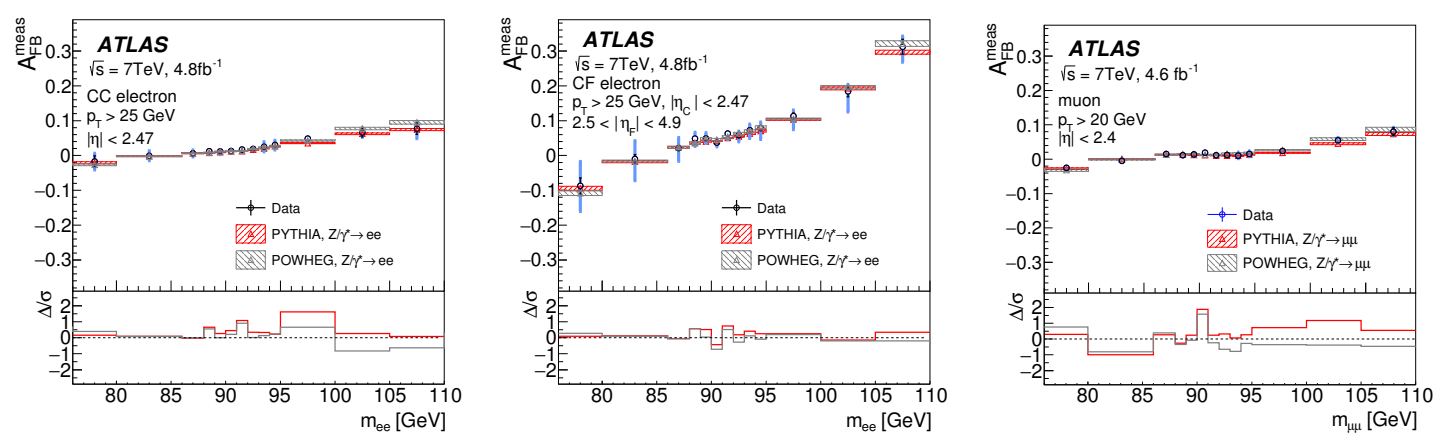

Figure 3: Detector-level forward-backward asymmetry values as a function of the dilepton invariant mass for the CC electron, $\mathrm{CF}$ electron and muon channels, after background subtraction. Data are shown by open circles and the the MC expectations is shown as a line [6].

One of the Standard Model parameters that can be extracted from the dependence of forwardbackward asymmetry on the invariant mass of dilepton system is the electroweak mixing angle $\sin ^{2} \theta_{\mathrm{W}}$. In this analysis, the value of the leptonic effective weak mixing angle $\sin ^{2} \theta_{\mathrm{eff}}^{\text {lept }}$ was extracted from each of the measured $A_{\mathrm{FB}}$ spectra by comparing the detector-level asymmetry to Monte Carlo predictions produced with varying initial values of the weak mixing angle (in the range 0.218 $\leq \sin ^{2} \theta_{\text {eff }}^{\text {lept }} \leq 0.238$ ). The templates were produced with PYTHIA [9] which allows tuning the value of $\sin ^{2} \theta_{\mathrm{eff}}^{\text {lept }}$ without changing the $m_{\mathrm{Z}}$. The generated templates are fitted to the data in the mass range $70-250 \mathrm{GeV}$ using a $\chi^{2}$ fit. For this measurement a special PDF set (ATLAS-epWZ12 LO) is used. This PDF set is a combination of ATLAS inclusive $W^{+}, W^{-}$and $Z$ production data at $\sqrt{s}=7 \mathrm{TeV}$ and combined HERA data on inclusive neutral- and charged-current interactions from $e^{+} p$ and $e^{-} p$ scattering $[10,11,12]$. The PDF uncertainty was estimated by reweighting to each of the eigenvalues in the ATLAS-epWZ12 LO PDF set at 68\% CL and then comparing to the central value [6]. The results for all three channels are shown in Table 1. The CF electron channel is the most precise because the dilution is the smallest. PDF uncertainty dominates the measurements, while the largest experimental uncertainty arises from the lepton energy scale.

The impact of the choice of different PDF sets is shown on Figure 4. The difference in the value of the $\sin ^{2} \theta_{\text {eff }}^{\text {lept }}$ due to PDF set used is comparable with the quoted PDF uncertainty. MSTW2008 set doesn't describe well the ATLAS $W$ and $Z$ data [11]. Further precision measurements are needed to constrain PDFs (inclusive $W / Z$ cross-section measurement, angular distributions).

Measured value $\sin ^{2} \theta_{\text {eff }}^{\text {lept }}=0.2308 \pm 0.0012$ is in agreement with PDG global fit $\sin ^{2} \theta_{\text {eff }}^{\text {lept }}=$ $0.23146 \pm 0.00012$. This result, which is limited by the PDF uncertainty, is about 10 times less precise than LEP+SLC [13]. A comparison of the results of this analysis with other published results for $\sin ^{2} \theta_{\text {eff }}^{\text {lept }}$ is shown on Figure 4. The muon asymmetry parameter $A_{\mu}$ can be estimated from $A_{\mathrm{FB}}, A_{\mu}=0.153 \pm 0.007$ (stat.) \pm 0.009 (syst.) $=0.153 \pm 0.012$ which is in agreement with measurements from LEP/SLD $0.142 \pm 0.015$ [13]. 


\begin{tabular}{l|c}
\hline \hline & $\sin ^{2} \theta_{\text {eff }}^{\text {lept }}$ \\
\hline \hline CC electron & $0.2302 \pm 0.0009$ (stat.) \pm 0.0008 (syst.) $\pm 0.0010(\mathrm{PDF})=0.2302 \pm 0.0016$ \\
CF electron & $0.2312 \pm 0.0007$ (stat.) \pm 0.0008 (syst.) $\pm 0.0010(\mathrm{PDF})=0.2312 \pm 0.0014$ \\
Muon & $0.2307 \pm 0.0009$ (stat.) \pm 0.0008 (syst.) $\pm 0.0009(\mathrm{PDF})=0.2307 \pm 0.0015$ \\
El. combined & $0.2308 \pm 0.0006$ (stat.) \pm 0.0007 (syst.) $\pm 0.0010(\mathrm{PDF})=0.2308 \pm 0.0013$ \\
\hline Combined & $0.2308 \pm 0.0005$ (stat.) \pm 0.0006 (syst.) $\pm 0.0009(\mathrm{PDF})=0.2308 \pm 0.0012$ \\
\hline \hline
\end{tabular}

Table 1: The $\sin ^{2} \theta_{\text {eff }}^{\text {lept }}$ measurement results in each of the three studied channels: electron central-central, electron central-forward and muon [6].
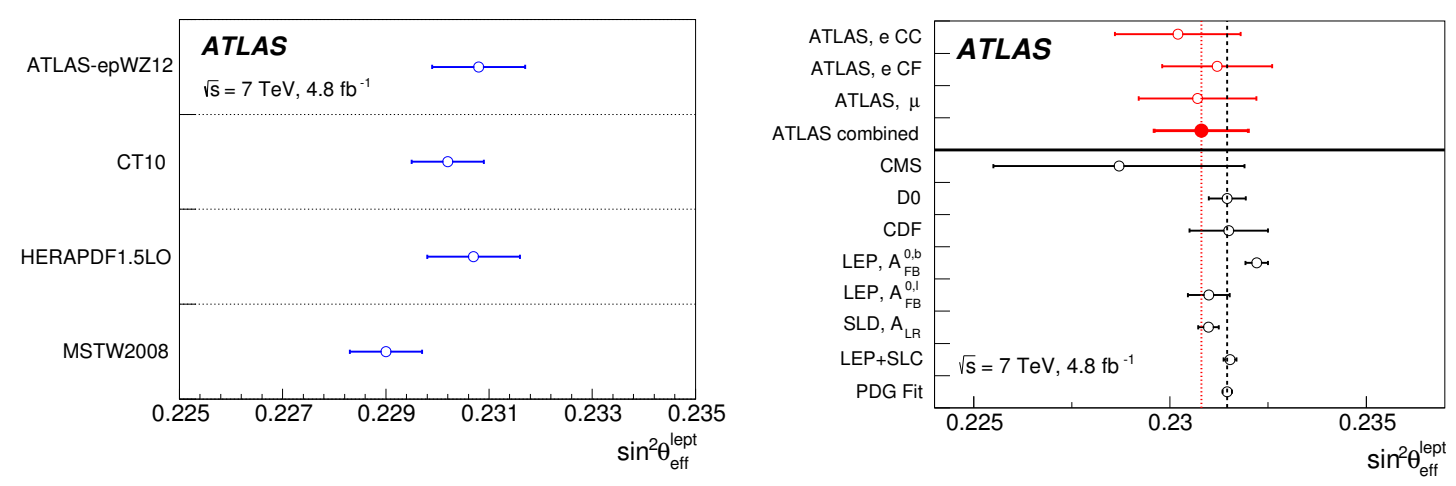

Figure 4: (a) Comparison of the extracted $\sin ^{2} \theta_{\text {eff }}^{\text {lept }}$ values when using different PDFs in the templates. Only PDF systematic errors are shown. (b) Comparison of the results of this analysis with other published results for $\sin ^{2} \theta_{\text {eff }}^{\text {lept }}[6]$.

\section{Preparation for the $m_{\mathrm{W}}$ measurement}

Among electroweak observables, the $W$ mass is the least constrained by current experimental data. Current world average value is $m_{\mathrm{W}}=80.385 \pm 0.015 \mathrm{GeV}$ which is including the most precise measurement form CDF and D0 experiments [14]. Indirect determination of $m_{\mathrm{W}}$ by electroweak fit is giving $\delta m_{\mathrm{W}}=8 \mathrm{MeV}$ [5] which is two times more accurate then the current measured value.

The $W$ boson mass is measured by comparing the data with MC templates of the mass-sensitive distributions: the transverse lepton momentum $p_{\mathrm{T}}^{1}$ and the transverse mass $m_{\mathrm{T}}$

$$
m_{\mathrm{T}}=\sqrt{2 p_{\mathrm{T}}^{1} p_{\mathrm{T}}^{v}(1-\cos \Delta \varphi(l, v))}
$$

where $p_{\mathrm{T}}^{v}$ is the transverse momentum of the neutrino and $\Delta \varphi(l, v)$ is the angle between the lepton and neutrino in the transverse plane. For the in-situ calibration of momentum and energy of the leptons $Z \rightarrow \ell \ell(\ell=e, \mu)$ events will be used. For the calculation of the missing transverse energy the hadronic recoil is used which is calibrated exploiting momentum balance in $Z$ events (after lepton calibration) $[15,16,17]$. The measurement is likely to be dominated by physics modeling (PDFs), therefore strong experimental constraints on the PDFs are needed to come from the $W$ cross sections, measured differentially in lepton pseudorapidity; in particular the $\eta$-dependent $W$ charge asymmetry is specifically sensitive to the $u$ and $d$ valence ratio. 
The current most precise measurement for $m_{\mathrm{W}}$ comes form Tevatron experiments with $\delta m_{\mathrm{W}}=$ $16 \mathrm{MeV}$ [18]. The dominating source of uncertainty is coming form the PDFs, the experimental systematic uncertainties are nearly purely of statistical origin [18]. The LHC measurement differs from the Tevatron in several respects: at the LHC the pile-up is higher which is affecting the hadronic recoil distribution, the proton-proton collisions compared to the proton-antiproton collision is giving different uncertainties from PDFs due to different contribution of valence and sea quarks. Furthermore, at the LHC there are high statistic calibration samples $(J / \psi, \Upsilon$ and $Z)$ which can be exploited for reduction of the experimental uncertainties. The ATLAS detector has a large number $W$ events in the data collected in Run- 1 available, which can be used to achieve $2 \mathrm{MeV}$ of statistical sensitivity.

\section{Conclusions}

The forward-backward asymmetry for the neutral current Drell-Yan process has been measured by the ATLAS experiment and found to be in agreement with the corresponding Standard Model predictions. The measured leptonic effective weak mixing angle $\sin ^{2} \theta_{\text {eff }}^{\text {lept }}=0.2308 \pm$ 0.0005 (stat.) \pm 0.0006 (syst.) \pm 0.0009 (PDF) $=0.2308 \pm 0.0012$ is in good agreement with the current world average from the Particle Data Group fit. The uncertainty is dominated by the PDFs. The determined value of muon asymmetry parameter $A_{\mu}=0.153 \pm 0.007$ (stat.) \pm 0.009 (syst.) $=$ $0.153 \pm 0.012$ is in agreement with previous measurements.

The ATLAS experiment has performed a wide range of measurements aimed at a better understanding of the the proton structure and the strong interaction, as well as detector calibration. To complete the measurement of $W$ boson mass further measurements are required in order to constrain the physics modeling uncertainties.

\section{References}

[1] ATLAS Collaboration, The ATLAS Experiment at the CERN Large Hadron Collider, JINST 3 S08003 (2008)

[2] ATLAS Collaboration, Observation of a new particle in the search for the Standard Model Higgs boson with the ATLAS detector at the LHC, Phys. Lett. B 716 (2012) 1, [hep-ex/1207 . 7214]

[3] CMS Collaboration, Observation of a new boson at a mass of $125 \mathrm{GeV}$ with the CMS experiment at the LHC, Phys. Lett. B 716 (2012) 30, [hep-ex/1207. 7235]

[4] ATLAS Collaboration, CMS Collaboration, Combined Measurement of the Higgs Boson Mass in pp Collisions at $\sqrt{s}=7 \mathrm{TeV}$ and $\sqrt{s}=8 \mathrm{TeV}$ with the ATLAS and CMS Experiments, Phys. Rev. Lett. 144 (2015) 191803, [hep-ex/1503.07589]

[5] The Gfitter Group, The global electroweak fit at NNLO and prospects for the LHC and ILC, Eur. Phys. J. C 74 (2014) 3046, [hep-ph/1407.3792]

[6] ATLAS Collaboration, Measurement of the forward-backward asymmetry of electron and muon pair-production in pp collisions at $\sqrt{s}=7 \mathrm{TeV}$ with the ATLAS detector, JHEP 09 (2015) 049, [hep-ex/1503 . 03709]

[7] J.C. Collins, D.E. Soper, Angular distribution of dileptons in high-energy hadron collisions, Phys. Rev. D 16 (1977) 2219

[8] G. D'Agostini, A multidimensional unfolding method based on Bayes' theorem, Nucl. Instrum. Meth. A 362 (1995) 487 
[9] T. Sjostrand, S. Mrenna, and P. Z. Skands, PYTHIA 6.4 physics and manual, JHEP 0605 (2006) 026, [hep-ph/0603175]

[10] ATLAS Collaboration, Determination of the strange quark density of the proton from ATLAS measurements of the $W \rightarrow l \mu$ and $Z \rightarrow$ ll cross sections, Phys. Rev. Lett. 109 (2012) 012001, [hep-ex/1203.4051]

[11] ATLAS Collaboration, Measurement of the inclusive $W^{ \pm}$and $Z / \gamma$ cross sections in the electron and muon decay channels in pp collisions at $\sqrt{s}=7 \mathrm{TeV}$ with the ATLAS detector, Phys. Rev. D 85 (2012) 072004, [hep-ex/1109.5141]

[12] F.D. Aaron et al., ZEUS, H1 collaboration, Combined measurement and QCD analysis of the inclusive $e^{ \pm} p$ scattering cross sections at HERA, JHEP 01 (2010) 109, [hep-ex/ 0911 . 0884 ]

[13] ALEPH, DELPHI, L3, OPAL, SLD, LEP Electroweak Working Group, SLD Electroweak Group, SLD Heavy Flavour Group Collaborations, Precision electroweak measurements on the Z resonance, Phys. Rept. 427 (2006) 257, [hep-ex/0509008]

[14] K.A. Olive et al., Particle Data Group, Chin. Phys. C 38 (2014) 010009

[15] ATLAS Collaboration, Measurement of the muon reconstruction performance of the ATLAS detector using 2011 and 2012 LHC proton-proton collision data, Eur. Phys. J. C 7411 (2014) [hep-ex/14 07 .3935]

[16] ATLAS Collaboration, Electron and photon energy calibration with the ATLAS detector using LHC Run 1 data, Eur. Phys. J. C 7410 (2014) [hep-ex/1407.5063]

[17] ATLAS Collaboration, Performance of missing transverse momentum reconstruction in proton-proton collisions at $\sqrt{s}=7 \mathrm{TeV}$ with ATLAS, Eur. Phys. J. C 721844 (2012) [hep-ex/1108.5602]

[18] T. Aaltonen et al., CDF and D0 Collaboration, Combination of CDF and DO W Boson Mass Measurements, Phys. Rev. D 88052018 (2013) [hep-ex/1307 . 7627] 